\title{
Towards a three-dimensional microfluidic liver platform for predicting drug efficacy and toxicity in humans
}

\author{
Abhinav Bhushan ${ }^{\dagger 1}$, Nina Senutovitch ${ }^{* 2,3}$, Shyam S Bale', William J McCarty', Manjunath Hegde', Rohit Jindal', \\ Inna Golberg', O Berk Usta', Martin L Yarmush', Lawrence Vernetti2,3, Albert Gough²,3, Ahmet Bakan³, Tong Ying Shun², \\ Richard DeBiasio² and D Lansing Taylor ${ }^{2,3}$
}

\begin{abstract}
Although the process of drug development requires efficacy and toxicity testing in animals prior to human testing, animal models have limited ability to accurately predict human responses to xenobiotics and other insults. Societal pressures are also focusing on reduction of and, ultimately, replacement of animal testing. However, a variety of in vitro models, explored over the last decade, have not been powerful enough to replace animal models. New initiatives sponsored by several US federal agencies seek to address this problem by funding the development of physiologically relevant human organ models on microscopic chips. The eventual goal is to simulate a human-on-a-chip, by interconnecting the organ models, thereby replacing animal testing in drug discovery and development. As part of this initiative, we aim to build a three-dimensional human liver chip that mimics the acinus, the smallest functional unit of the liver, including its oxygen gradient. Our liver-on-a-chip platform will deliver a microfluidic three-dimensional co-culture environment with stable synthetic and enzymatic function for at least 4 weeks. Sentinel cells that contain fluorescent biosensors will be integrated into the chip to provide multiplexed, real-time readouts of key liver functions and pathology. We are also developing a database to manage experimental data and harness external information to interpret the multimodal data and create a predictive platform.
\end{abstract}

\section{${ }^{+}$Contributed equally.}

*Correspondence: ninas@pitt.edu

${ }^{2}$ Drug Discovery Institute, University of Pittsburgh, 10040 Biomedical Science

Tower 3, 3501 Fifth Avenue, Pittsburgh, PA 15260, USA

Full list of author information is available at the end of the article

\section{Introduction}

The liver is the largest metabolically active organ and is important in the modification and detoxification of external agents, but is also susceptible to damage from these substances [1] and their metabolic intermediates. Liver toxicity and cardiotoxicity are the most cited reasons for both market withdrawal and failure during late-stage clinical testing of drugs [2,3]. Current animal models, as well as in vitro liver platforms, are poor predictors of human liver toxicities, with success rates as low as $40 \%[4,5]$. The pharmaceutical industry is therefore in need of better tools for predicting these toxicities in early stages of development in order to reduce dangerous clinical outcomes and drug development costs. Thus in 2011 the National Institutes of Health, through the National Center for Advancing Translational Science, the US Food and Drug Administration, the Environmental Protection Agency and the Defense Advanced Research Projects Agency, announced a collaboration to develop microphysiological systems that model major organs of the human body. Key requirements for these models are: to facilitate the assessment of biomarkers, bioavailability, efficacy, and toxicity of therapeutic agents prior to clinical trials; and to predict the safety, efficacy, and pharmacokinetics of drug/vaccine candidates prior to their first human use.

As participants in the National Center for Advancing Translational Science program, our laboratories at the University of Pittsburgh and at Massachusetts General Hospital are collaboratively developing a three-dimensional microfluidic human liver acinus with integrated fluorescent biosensors to improve prediction of human liver response to xenobiotic insults (Figure 1). Fluorescence-based protein biosensors can detect altered levels of specific analytes and changes of subcellular protein localization with spatiotemporal resolution in response to drugs or external stimuli [6,7]. The platform comprises four vital cell types of the liver (hepatocytes, endothelial, stellate and Kupffer cells) arranged layer by layer with 


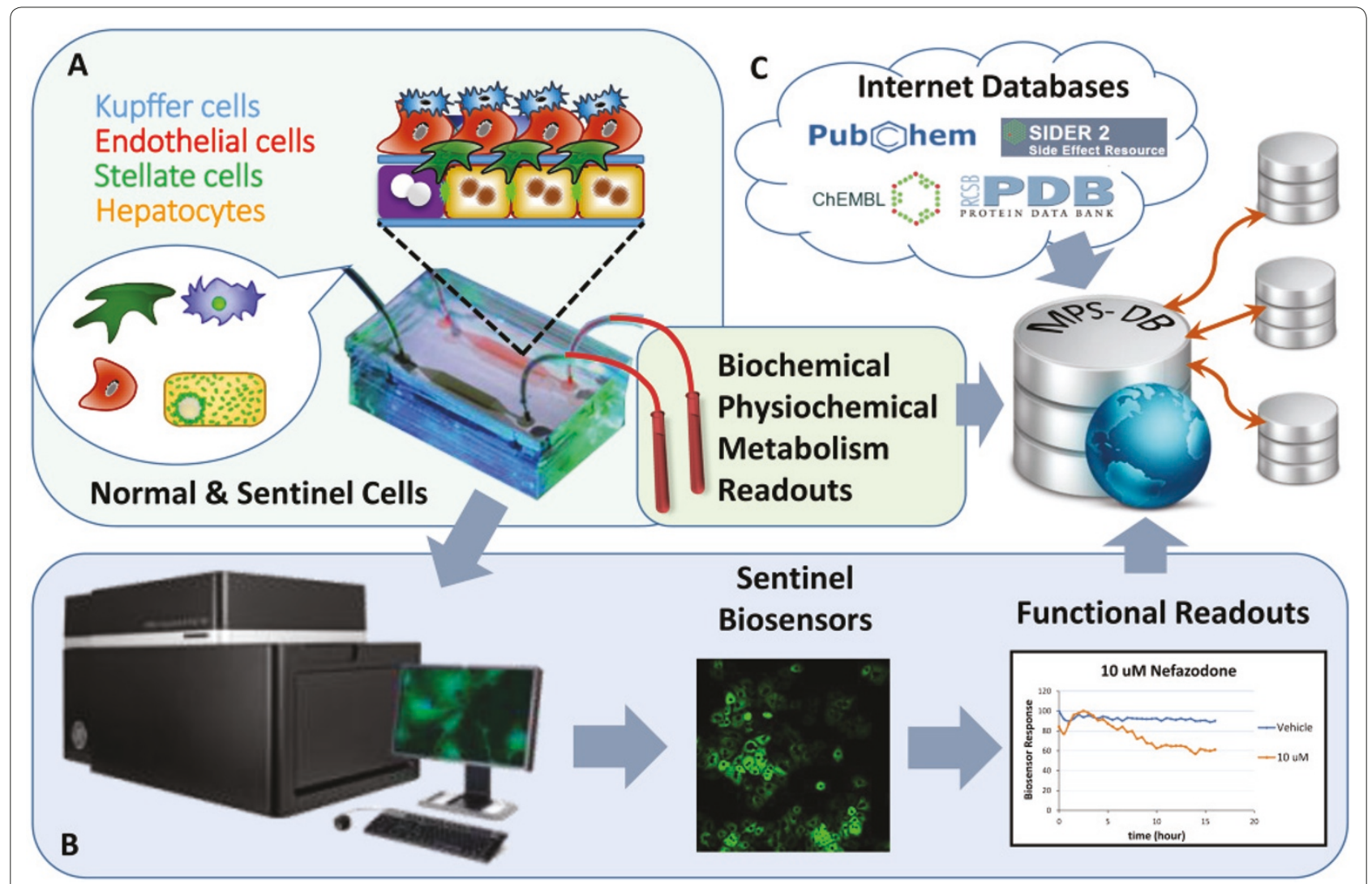

Figure 1. Liver acinus module with microchip. (A) The liver acinus module with a microchip, including a diagram of four liver cell types and sentinel biosensor cells layered in the device. (B) High content analysis of microchip device and quantitation. (C) Graphic depiction of data from device and external sources supplied to database.

well-defined cell numbers and organization in a threedimensional microfluidic environment mimicking a hepatic cord. A subset of hepatocytes and nonparenchymal cells (NPC) integrated into the device are biosensor sentinel cells, expressing fluorescence-based biosensors of key cellular functions in order to provide quantitative, real-time reports of cell health and molecular modes of action [8]. This approach seeks to extend the predictive relationship between hepatotoxicity triggers (mitochondrial damage, activation of Kupffer cell, oxidative stress) and the final manifestation of drug-induced liver injury (DILI) to long-term and real-time dynamics [9]. Since DILI can manifest as both acute and chronic effects, our liver platform is being developed to function for at least 4 weeks; a significant technical challenge considering the rapid de-differentiation usually observed for primary cell cultures. Another key component of our approach is the development of a database application that will access information from major drugs, drug targets, bioassays and pathway databases. Related chemical, bioactivity, preclinical and clinical data will be used for aiding the interpretation of microphysiology readouts and development of computational models to predict the safety and toxicity of new compounds. The database design aim is ultimately to integrate the other microphysiological organ systems to complete a human-on-a-chip database.

\section{Capturing precise liver physiology in vitro}

The liver comprises two major cell populations: parenchymal cells (hepatocytes) and NPCs, including endothelial cells, stellate cells and Kupffer cells among others. The inspiration for our platform is the liver sinusoid and the acinus, the smallest metabolic functional unit of the liver. There are four crucial aspects of liver physiology captured in our design: a three-dimensional architecture; multiple cell types; physiological microcirculation; and zonation.

A model that aims to recapitulate the intricate autocrine and paracrine interaction between different cells of the liver should include the major parenchymal cell and NPC types as well as a three-dimensional microcellular environment mimicking the acinus-sinusoid [10]. The three-dimensional architecture shaped by the collagen sandwich in tissue culture plates was an important development in the long-term stable metabolic and synthetic function of hepatocytes [11]. Co-cultures of 
liver cells can extend the stability and function even further, as well as modify the response to drugs as compared with monocultures of hepatocytes [12-14]. Kupffer cells, for example, are known to contribute to DILI during acetaminophen toxicity [15]. These cocultures can also stabilize cells after unavoidable injury [13]. Recent results show that both human and rat hepatocytes are stable for over 3 months in a co-culture with their respective NPC fraction, with improved response to drug induction of clinically relevant cytochrome P450 enzymes [12], highlighting the importance of a coculture over standard monocultures. Further, arranging the co-cultures into sinusoid-like structures improves albumin secretion and cytochrome P450 activity for over 2 months [16]. The latter is important as evidence suggests reactive metabolites are often the cause of unanticipated DILI. Evidence further confirms that threedimensional co-cultures, either on a scaffold or as a spheroid, improve both the longevity and toxicological response [12,17,18].

Microcirculatory flow can also modulate the response of hepatocytes [19] and of endothelial cells [20], and can form the oxygen zonation that is important for site-directed injury and the transport of nutrients and waste metabolites. However, when exposing cells to flow, it is important to emulate physiologically relevant conditions including appropriate shear stress, drug residence times, and chemical gradients. The microfluidic approaches allow for such emulation as well as providing high-throughput test beds for pharmacokinetic and toxicity studies.

\section{Modes of action to predict human hepatoxicity and approach}

The exact molecular basis for many instances of DILI is unknown. A major goal of our project is to identify realtime functional responses associated with hepatotoxicity using sentinel cells [21]. We are developing the sentinel cells of various cell types to respond to several molecular signatures that are associated with hepatotoxicity [22]. Based on our previous experience with biosensors, we are developing hepatocyte sentinel cells to report metabolic alterations due to apoptosis, oxidative stress, phospholipidosis, and intrahepatic cholestasis caused by inhibition of bile efflux transport, among others [7,23,24]. Kupffer cell activation in response to the injury trigger will be used to measure immune dysfunction that occurs as a result of reaction to a drug, a drug metabolite, or a protein altered by a reactive drug metabolite [25]. Stellate cells will have biosensors to monitor proliferation that results from fibrosis associated with cholestasis or mitochondrial failure [26]. Cells placed in the chip are also confirmed for intermediary metabolism.

Our vision is to use a microfluidic device where the hepatic and sentinel cells are arranged in parallel microgrooves in a sinusoid-like architecture (Figure 2A,B,C) [27]. The cells at the inlet of these channels consume oxygen, which generates an oxygen gradient along the flow direction of the device. Previous work on microfluidic liver(s)-on-a-chip used feeder cells to stabilize cultures, but lacked long-term functional data and had no incorporation of NPCs in a three-dimensional architecture [28-30]. Although bioreactors include longer culture times and multiple cell types, they are not suited for microfluidic format [31]. Our strategy is to seed the hepatocytes and add the NPCs sequentially. The cells will be proportioned in appropriate physiological ratios of 70 to $80 \%$ hepatocytes, 15 to $20 \%$ endothelial cells, 5 to $10 \%$ stellate cells and 5 to $10 \%$ Kupffer cells.

Our initial prototype for cell culture and parameter optimization is a cell culture chamber $10 \mathrm{~mm}$ long, $1.5 \mathrm{~mm}$ wide, and $100 \mu \mathrm{m}$ tall, fabricated out of polydimethylsiloxane [32]. In preliminary work, approximately 12,000 seeded hepatocytes demonstrated stable hepatocyte functionality for 10 days of culture under a static (no-flow) condition, showing excellent viability (Figure 2D,E). Although the device incorporates human cells, preliminary device optimization was carried out using primary rat liver cells. Similarly, polydimethylsiloxane used in prototypes will be replaced with more robust materials. The functional data are supported by periodic cell viability assays as well as visualization of the intracellular albumin (Figure 2F).

A novel concept in our vision is to integrate multiple live, stable, and nondestructive cellular reporters of test drug effects to enable the early prediction of DILI using high content analysis. A subset of primary human liver cells is efficiently transformed into sentinel cells by lentiviral gene delivery of fluorescent protein biosensors. Long-term high content analysis can be carried out within the microfluidic device without the need for offline analysis [33] (Figure 1). The sentinel cell data are quantifiable to assess the toxicity, metabolic functions, and key modes of action responses to drugs over time. Initial studies of sentinel cell primary human hepatocytes report increased reactive oxygen species (Figure 2FG) and mitochondrial impairment (Figure $2 \mathrm{H}, \mathrm{I}$ ) in response to known hepatotoxins versus a vehicle control. Importantly, these responses are time and concentration dependent, reproducible, quantitative and collected by nondestructive techniques. Sentinel cell data will be integrated with biochemical and metabolic measurements to monitor drug clearance and cytochrome P450 induction, glucose consumption, and urea and albumin secretions. The sentinel cell concept will advance the field because it enables the study of primary cell heterotypic interactions due to drug response and enables the study of dynamic cellular response from within a functional liver model. 

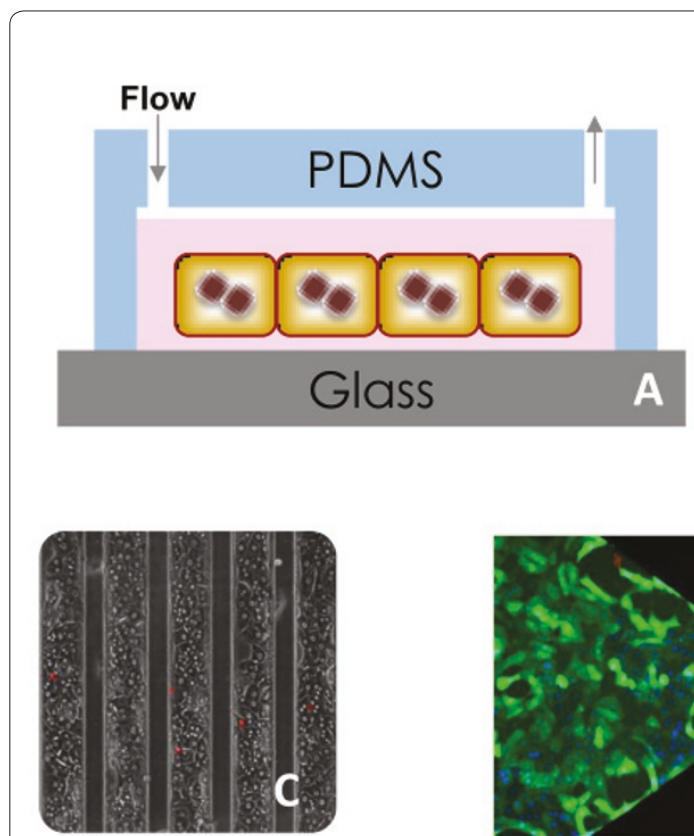

\section{Hepatocytes in} microgrooves

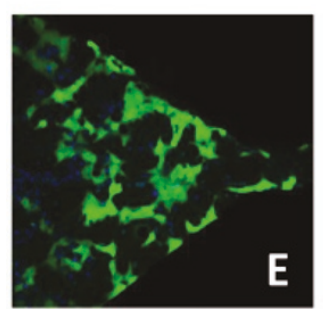

Day 9

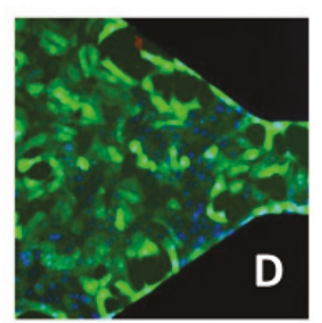

Day 3

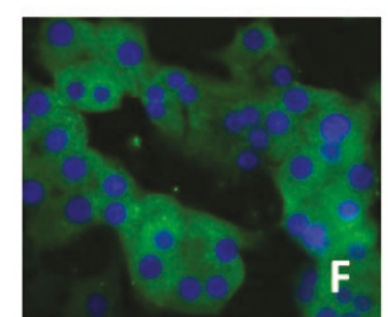

Intracellular Albumin

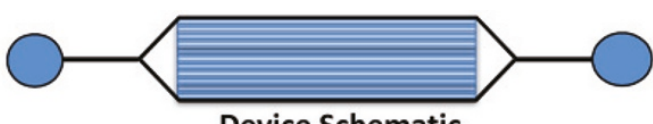

Device Schematic

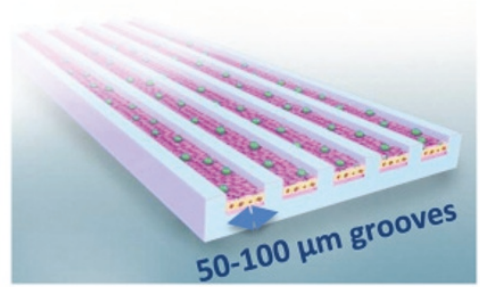

B

Micro-grooved design to create liver sinusoids
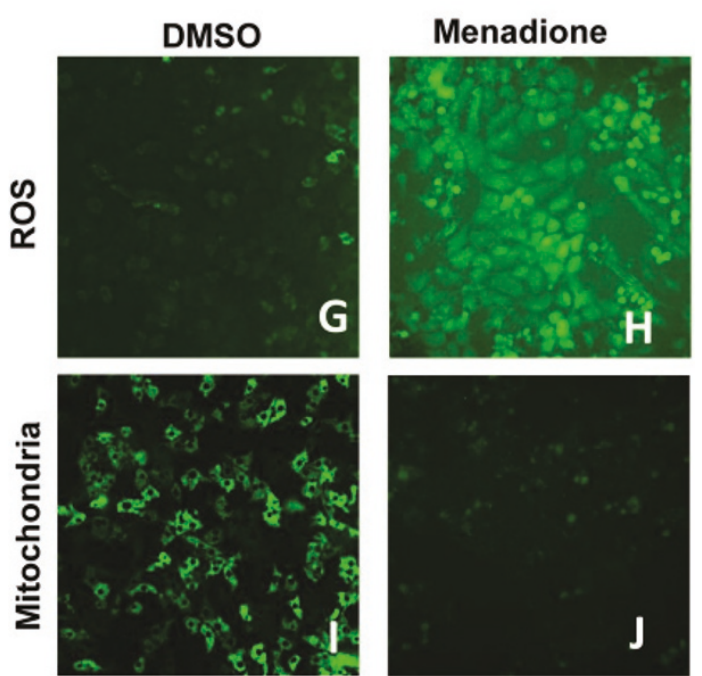

Figure 2. Preliminary results. (A) Diagram of flow conditions for hepatocytes in collagen gel in the microfluidic device. PDMS, polydimethylsiloxane. (B) Microfluidic channels $50 \mu \mathrm{m}$ wide, $10 \mathrm{~mm}$ long, and $100 \mu \mathrm{m}$ tall with grooves to represent a liver sinusoid. (C) Phase image of primary hepatocytes in a microgrooved channel [27]. (D), (E) Live primary rat hepatocytes stained for live (celcein)/dead (ethidium homodimer-1) cells at days 3 and 9. (F) Fixed primary rat hepatocytes stained for intracellular albumin (anti-rat albumin antibody; Abcam, Cambridge, MA, USA). (G), (H) Primary human hepatocyte sentinel cells expressing reactive oxygen species (ROS) biosensor treated with dimethylsulfoxide (DMSO; control) or $100 \mu \mathrm{mol}$ menadione for approximately 4 hours. (I), (J) Primary human hepatocyte sentinel cells expressing mitochondrial function biosensor treated with DMSO (control) or $100 \mu \mathrm{mol}$ menadione, approximately 16 hours after addition of treatments.

Central to the liver model, and indeed all of the organ models, is the organization and analysis of large and complex datasets to predict human organ efficacy and safety [21]. We are constructing a database application to collect, manage, and analyze data from the real-time readouts, biochemical assays, and mass spectrometry. Beyond data management, the database will incorporate access to clinically relevant hepatotoxic and nonhepatotoxic drug exposure results, and biochemical and metabolic activities in order to establish the concordance of the liver chip data with in vivo effects. To achieve this goal, a selection of reference compounds with available clinical data will be profiled in the liver chip, and the resulting dataset will be used to construct predictive models of human hepatotoxicity.

\section{Conclusions and future directions}

We are particularly interested in using the liver acinus module to investigate DILI, which leads to pathologies such as fibrotic scarring, fatty liver disease, reduced metabolic detoxification, and even liver failure [1]. An important cause of DILI is human genetic variability that can lead to altered liver metabolism and response to drugs. Our long-term goal is to seed the liver microchip with induced pluripotent stem cell-derived adult-like hepatocytes to characterize and predict hepatotoxicity 
arising from distinct genetic backgrounds. Since our liver acinus module is modular and reconfigurable, we plan to combine it with other organ platforms to begin assessing multiorgan toxicities. Key elimination organs that have direct interaction with the liver, such as the gut and kidney, are of particular importance because they can provide robust pharmacokinetic modeling. This plan fits our goals of: generating a microscale platform technology to support human physiological organ systems; maintaining the liver module at high, stable levels of function for at least 4 weeks; and utilizing real-time high content measurements and a reference database to improve the prediction of human liver toxicity. Finally, sentinel biosensor cells can be developed for other organs and the general design of our database application is adaptable for other organ models.

\section{Abbreviations}

DILI, drug-induced liver injury; NPC, nonparenchymal cell.

\section{Competing interests}

The authors declare that they have no competing interests.

\section{Acknowledgements}

This work was supported in part by the National Institutes of Health Common Fund 1 UH2TR000503 (to DLT and MLY, co-principal investigators). Publication costs are funded by the National Institutes of Health, National Center for Advancing Translations Science under grant 5UH2TR000503-02 to DLT.

\section{Declarations}

Publication of this supplement has not been supported by sponsorship. Articles have undergone the journal's standard review process. The Editors declare that they have no competing interests.

This article has been published as part of Stem Cell Research \& Therapy Volume 4 Supplement 1, 2013: Stem cells on bioengineered microphysiological platforms for disease modeling and drug testing. The full contents of the supplement are available online at http://www.stemcellres.com/supplements/4/S1.

\section{Author details}

${ }^{1}$ Center for Engineering in Medicine at Massachusetts General Hospital, Harvard Medical School, Shriners Hospital for Children at Boston, Boston, MA 02114, USA. 2Drug Discovery Institute, University of Pittsburgh, 10040 Biomedical Science Tower 3, 3501 Fifth Avenue, Pittsburgh, PA 15260, USA. 32Department of Computational \& Systems Biology, University of Pittsburgh, Pittsburgh, PA 15260, USA.

Published: 20 December 2013

\section{References}

1. Lee WM, Senior JR: Recognizing drug-induced liver injury: current problems, possible solutions. Toxicol Pathol 2005, 33:155-164.

2. Wilke RA, Lin DW, Roden DM, Watkins PB, Flockhart D, Zineh I, Giacomini KM, Krauss RM: Identifying genetic risk factors for serious adverse drug reactions: current progress and challenges. Nat Rev Drug Discov 2007, 6:904-916.

3. Laverty H, Benson C, Cartwright E, Cross M, Garland C, Hammond T, Holloway C, McMahon N, Milligan J, Park B, Pirmohamed M, Pollard C, Radford J, Roome N, Sager P, Singh S, Suter T, Suter W, Trafford A,Volders P, Wallis R, Weaver R, York $M$, Valentin J: How can we improve our understanding of cardiovascular safety liabilities to develop safer medicines? Br J Pharmacol 2011, 163:675-693.

4. Greaves P, Williams A, Eve M: First dose of potential new medicines to humans: how animals help. Nat Rev Drug Discov 2004, 3:226-236.
5. Olson H, Betton G, Robinson D, Thomas K, Monro A, Kolaja G, Lilly P, Sanders J, Sipes G, Bracken W, Dorato M, Van Deun K, Smith P, Berger B, Heller A: Concordance of the toxicity of pharmaceuticals in humans and in animals. Regul Toxicol Pharmacol 2000, 32:56-67.

6. Morris MC: Fluorescent biosensors - probing protein kinase function in cancer and drug discovery. Biochim Biophys Acta 2013, 1834:1387-1395.

7. Giuliano KA, Post PL, Hahn KM, Taylor DL: Fluorescent protein biosensors: measurement of molecular dynamics in living cells. Annu Rev Biophys Biomol Struct 1995, 24:405-434.

8. Albrecht SC, Sobotta MC, Bausewein D, Aller I, Hell R, Dick TP, Meyer AJ: Redesign of genetically encoded biosensors for monitoring mitochondrial redox status in a broad range of model eukaryotes. J Biomol Screen 2013. [Epub ahead of print]

9. Xu JJ, Henstock PV, Dunn MC, Smith AR, Chabot JR, de Graaf D: Cellular imaging predictions of clinical drug-induced liver injury. Toxicol Sci 2008, 105:97-105.

10. Moslen MT: Toxic responses of the liver. In Casarett and Doull's Toxicology The Basic Science of Poisons. 5th edition. Edited by Klaasen CD, Doull J. New York: McGraw Hill; 1996:403-416.

11. Dunn JC, Tompkins RG, Yarmush ML: Long-term in vitro function of adult hepatocytes in a collagen sandwich configuration. Biotechnol Prog 1991, 7:237-245.

12. Kostadinova R, Boess F, Applegate $D$, Suter L, Weiser T, Singer T, Naughton B, Roth A: A long-term three dimensional liver co-culture system for improved prediction of clinically relevant drug-induced hepatotoxicity. Toxicol Appl Pharmacol 2013, 268:1-16.

13. Jindal R, Nahmias Y, Tilles AW, Berthiaume F, Yarmush ML: Amino acidmediated heterotypic interaction governs performance of a hepatic tissue model. FASEB J 2009, 23:2288-2298.

14. Bhatia SN, Balis UJ, Yarmush ML, Toner M: Effect of cell-cell interactions in preservation of cellular phenotype: cocultivation of hepatocytes and nonparenchymal cells. FASEB J 1999, 13:1883-1900.

15. Ju C, Reilly TP, Bourdi M, Radonovich MF, Brady JN, George JW, Pohl LR Protective role of Kupffer cells in acetaminophen-induced hepatic injury in mice. Chem Res Toxicol 2002, 15:1504-1513.

16. Nahmias Y, Schwartz RE, Hu WS, Verfaillie CM, Odde DJ: Endotheliummediated hepatocyte recruitment in the establishment of liver-like tissue in vitro. Tissue Eng 2006, 12:1627-1638.

17. Drewitz M, Helbling M, Fried N, Bieri M, Moritz W, Lichtenberg J, Kelm JM: Towards automated production and drug sensitivity testing using scaffold-free spherical tumor microtissues. Biotechnol J 201 1, 6:1488-1496.

18. Hwa AJ, Fry RC, Sivaraman A, So PT, Samson LD, Stolz DB, Griffith LG: Rat liver sinusoidal endothelial cells survive without exogenous VEGF in 3D perfused co-cultures with hepatocytes. FASEB J 2007, 21:2564-2579.

19. Cho CH, Park J, Nagrath D, Tilles AW, Berthiaume F, Toner M, Yarmush ML: Oxygen uptake rates and liver-specific functions of hepatocyte and 3T3 fibroblast co-cultures. Biotechnol Bioeng 2007, 97:188-199.

20. Shay-Salit A, Shushy M, Wolfovitz E, Yahav H, Breviario F, Dejana E, Resnick N: VEGF receptor 2 and the adherens junction as a mechanical transducer in vascular endothelial cells. Proc Natl Acad Sci U S A 2002, 99:9462-9467.

21. Vernetti L, Irwin W, Giuliano KA, Gough A, Johnston K, Taylor DL: Cellular systems biology applied to preclinical safety testing: a case study of CellCiphr ${ }^{\mathrm{TM}}$ profiling. In Drug Efficacy, Safety, and Biologics Discovery: Emerging Technologies and Tools. Edited by Ekins S, Xu JJ. Hoboken, NJ: Wiley \& Sons; 2009:53-73.

22. Jaeschke H, Gores GJ, Cederbaum Al, Hinson JA, Pessayre D, Lemasters JJ: Mechanisms of hepatotoxicity. Toxicol Sci 2002, 65:166-176.

23. Giuliano KA, Gough AH, Taylor DL, Vernetti LA, Johnston PA: Early safety assessment using cellular systems biology yields insights into mechanisms of action. J Biomol Screen 2010, 15:783-797.

24. Giuliano KA, Taylor DL, Waggoner AS: Reagents to measure and manipulate cell functions. Methods Mol Biol 2007, 356:141-163.

25. Holt MP, Ju C: Mechanisms of drug-induced liver injury. AAPS J 2006, 8:E48-E54

26. Guicciardi ME, Gores GJ: Apoptosis as a mechanism for liver disease progression. Semin Liver Dis 2010, 30:402-410

27. Park J, Berthiaume F, Toner M, Yarmush ML, Tilles AW: Microfabricated grooved substrates as platforms for bioartificial liver reactors. Biotechnol Bioeng 2005, 90:632-644.

28. Lee PJ, Hung PJ, Lee LP: An artificial liver sinusoid with a microfluidic endothelial-like barrier for primary hepatocyte culture. Biotechnol Bioeng 
2007, 97:1340-1346.

29. Bhatia SN, Balis UJ, Yarmush ML, Toner M: Microfabrication of hepatocyte/ fibroblast co-cultures: role of homotypic cell interactions. Biotechnol Prog 1998, 14:378-387.

30. Mockel GM, Gorti S, Tandon RK, Tanaka T, Carey MC: Microscope laser lightscattering spectroscopy of vesicles within canaliculi of rat hepatocyte couplets. Am J Physiol 1995, 269:G73-G84.

31. Tan GD, Toh GW, Birgersson E, Robens J, van Noort D, Leo HL: A thin-walled polydimethylsiloxane bioreactor for high-density hepatocyte sandwich culture. Biotechnol Bioeng 2013, 110:1663-1673.

32. McDonald JC, Whitesides GM: Poly(dimethylsiloxane) as a material for fabricating microfluidic devices. Acc Chem Res 2002, 35:491-499.
33. Giuliano KA, Premkumar D, Taylor DL: Optimal characteristics of proteinprotein interaction biosensors for cellular systems biology profiling. In High Content Screening: Science, Techniques and Applications. Edited by Haney SA. Hoboken, NJ: Wiley-Interscience; 2008:371-387.

doi:10.1186/scrt377

Cite this article as: Bhushan A, et al.: Towards a three-dimensional microfluidic liver platform for predicting drug efficacy and toxicity in humans. Stem Cell Research \& Therapy 2013, 4(Suppl 1):S16. 